\title{
Do (non-American) Men Overestimate Women's Sexual Intentions?
}

\author{
Carin Perilloux ${ }^{1}$ • José Antonio Muñoz-Reyes ${ }^{2} \cdot$ Enrique Turiegano $^{3}$. \\ Robert Kurzban $^{4} \cdot$ Miguel Pita $^{3}$
}

Published online: 18 April 2015

(C) Springer International Publishing 2015

\begin{abstract}
Prior research has suggested that men overestimate women's sexual intentions. However, the bulk of the data supporting this view comes from participants from the USA. Here, we report three attempts to replicate this effect in samples from Chile, Spain, and France. While there was some evidence of overestimation of sexual intent by men on the aggregate measure, removing a single item decreases or even eliminates the sex difference in some of the cultures studied, suggesting that the aggregate effect is driven by a small number of particular behaviors. Furthermore, women from the USA appear to rate sexual intent differently from men and women in the other countries, whose ratings are relatively more homogeneous. While more work is needed, these results raise the possibility that the sex differences in sexual intent perception documented in the USA might not be crossculturally universal.
\end{abstract}

Keywords Sexual intent perception $\cdot$ Cross-cultural $\cdot$ Sex differences $\cdot$ Spain $\cdot$ Chile $\cdot$ France

Carin Perilloux

carinp@gmail.com

1 Department of Psychology, Texas State University, 601 University Drive, San Marcos, TX 78666, USA

2 Centro de Estudios Avanzados, Universidad de Playa Ancha, Valparaíso, Chile

3 Departamento de Biología, Universidad Autónoma de Madrid, Madrid, Spain

4 Department of Psychology, University of Pennsylvania, Philadelphia, PA 19104, USA
For decades, psychologists have gathered evidence that men appear to overestimate women's sexual interest in them. Since Abbey's (1982) original study, researchers have used various methods that converge on the conclusion that men infer more sexual intent on the part of women than women self-report. Men's estimates of women's underlying sexual intentions are consistently higher than women's own reports in the lab, on self-report surveys, and when viewing photographs or videos (see Farris et al. 2008, for a review).

To explain this pattern, error management theory (EMT) applies a decision theory approach to the evolution of cognitive biases, a bias in men toward inferring greater sexual interest in this case (Haselton and Buss 2000). EMT asserts that cognitive biases can evolve when the costs of one type of error (either a miss or false alarm) have been recurrently larger than the costs of the other type of error. In the context of sexual intent perception, EMT begins with the assumption that, for men, the costs of misses are much larger than the costs of false alarms: a man who consistently missed out on sexually interested women presumably experienced a much larger loss in the currency of reproductive success (i.e., potential reproduction) than a man who tended to commit false alarms in thinking that women were more interested in him than they actually were (e.g., wasted time and effort). This difference would have held, on average, throughout evolutionary history, and therefore, EMT predicts that selection would favor cognitive biases in men that cause them to infer more sexual interest on the part of women rather than less.

In the original EMT study of sexual intent perception (Haselton and Buss 2000), men did report that various dating behaviors engaged in by a hypothetical woman would mean she had higher sexual intentions than those same behaviors rated by women themselves. Notably, the researchers found that this effect disappeared when men were assessing the behaviors of a sister on a date with someone as opposed to their 
own potential date, indicating that men do not invariably assume greater sexuality on the part of women. Indeed, subsequent studies of sexual interest perception indicate that the effect can be influenced by the attractiveness of the target female (Perilloux et al. 2012), the man's interest in casual sex (Howell et al. 2012; Perilloux et al. 2012), and cues of sexual accessibility such as revealing clothing (Abbey et al. 1987).

Context effects and individual differences notwithstanding because the reproductive consequences of misses and false alarms were recurrent for men, the proposed adaptive cognitive bias should be present cross-culturally (though perhaps expressed to varying degrees, depending on circumstances or individual traits). This implication of the hypothesis (i.e., universality) has not yet been tested, however. Thus far, only two studies that we are aware of have attempted to explicitly document men's overestimation cross-culturally. DeSouza et al. (1992) provided hypothetical dating scenarios to men and women in Brazil and in the USA. They found the typical large and significant sex difference in USA; in Brazil, the sex difference - though in the expected direction - was not significant. Bendixen (2014), in contrast, did replicate in a Norwegian sample the tendency for women to report having their sexual intent overestimated by men more than underestimated.

The goal of the current study was to assess whether the sex difference would replicate across multiple cultures. We used the same method as the original EMT study (Haselton and Buss 2000) with samples from Spain, Chile, and France.

\section{Method}

\section{Participants}

We collected samples of participants from Spain, Chile, and France. For the samples from Spain and France, we recruited students, friends, and family on a volunteer basis and they were encouraged to share the link to the online questionnaire via a snowball collection procedure. In Chile, we recruited volunteers from a database of postgraduates of a large university. We removed homosexual participants from each sample: six from the Spanish sample, five from the Chilean sample, and five from the French sample. The final sample from Spain included 102 women and 76 men with a mean age of 31.30 years $(\mathrm{SD}=8.95){ }^{1}{ }^{1}$ The final sample from Chile included 47 women and 54 men with a mean age of 32.80 years $(\mathrm{SD}=7.62)$. The final sample from France included 56 women and 37 men with a mean age of $31.32(\mathrm{SD}=6.46)$.

\footnotetext{
${ }^{1}$ Ages ranged from 19 to 80 years in the Spanish sample, 19 to 60 years in the Chilean sample, and 18 to 57 years in the French sample.
}

\section{Materials}

Participants completed the sexual intent ratings from the dating behaviors scale (DBS; Haselton and Buss 2000) which was translated $^{2}$ into Spanish and French. Women rated their likelihood of having sex with a man they had been on a few dates with based on having engaged in each of 15 behaviors (from -3 "extremely unlikely" to +3 "extremely likely"). Men used the same scale to estimate a hypothetical woman's likelihood of having sex with them after having been on a few dates, given that she engaged in each of the 15 behaviors. Items were in the form, "If [I/she] [behavior], what is the likelihood [I/she] would want to have sex?" The behaviors listed in the scale were (with pronouns adjusted appropriately): held hands with me, bought me expensive jewelry, complimented me on my appearance, treated me to an expensive dinner, passionately kissed me, had sex with me, sent me a dozen red roses, told me that she loved me, told me that she would like to spend her life with me, stared deeply into my eyes when we were out on a date, cooked me a gourmet dinner, put her hand on my thigh, told me she would like to meet my family, told me that she felt like she had known me forever, suggested that we go back to her apartment for a drink. The scale demonstrated good reliability in each sample: Cronbach's $\alpha=.86$ (Spain), Cronbach's $\alpha=.85$ (Chile), Cronbach's $\alpha=.83$ (France). A composite rating for the scale was calculated as the average of a participant's responses to all 15 items.

\section{Procedure}

Participants followed a link provided to the online survey hosted by Google documents. They first answered demographic questions then proceeded to complete the DBS.

\section{Results}

We used data from Perilloux and Kurzban (2015) to serve as our USA comparison group because it was collected recently and because we had access to the raw data which were useful in conducting the cross-cultural evaluations presented below.

Independent $t$ tests revealed the overall sex difference in the composite ratings replicated in each of our samples, with men providing significantly higher ratings than women, Spain,

\footnotetext{
${ }^{2}$ For the Spanish version, two researchers from Spain (MP and ET) and one researcher from Chile (JM) independently translated the scale and then met to discuss the translations and agreed upon a consensus version. This was back-translated to English by a bilingual Spanish speaker unaffiliated with the project to confirm accuracy. For the French version, one of us (MP) translated the instrument to French and then a bilingual French speaker who was unaffiliated with the project back-translated the instrument to confirm accuracy.
} 
$t(176)=4.30, p<.001, d=0.66$; Chile, $t(99)=2.52, p=.01$, $d=0.50$; France, $t(91)=2.24, p=.03, d=0.50$. Figure 1 depicts these sex differences alongside the sex difference documented in the USA, $t(482)=7.49, p<.001, d=0.66$.

In fact, when we compare all of the groups separately (USA women, USA men, Spanish women, Spanish men, Chilean women, Chilean men, French women, and French men), $F(7$, $848)=16.49, p<.001, \eta_{\mathrm{p}}{ }^{2}=.12$, we find that USA women score significantly lower than all the other groups except women from Chile (Tukey's HSD $p<.05$ for all comparisons). None of the other pairwise comparisons was significant except that Spanish men had higher ratings than Spanish women (Tukey's HSD $p<.01$ ) and Chilean women (Tukey's HSD $p=.01$ ).

Examining the individual items of the scale shows a different pattern, however. As displayed in Table 1, there were many more significant sex differences by item in the USA sample than in the samples from Spain, Chile, or France. The sex differences in the composites might be driven by outlier items showing a profound difference. For example, the item Bought Jewelry shows the largest sex difference and in fact appears to be rated differently from the other items by women in all non-USA samples. If we remove Bought Jewelry from the composite scores, the composite sex differences in the Chilean, $t(99)=1.89, p=.06, d=0.38$, and French, $t(91)=1.97, p=.12, d=0.12$, samples drop below the threshold for significance; the Spanish sample still exhibits a significant sex difference, though it is much smaller, $t(176)=3.63$, $p<.001, d=0.56$, and the USA sample also shows a small decrease in effect size, $t(482)=6.92, p<.001, d=0.61$.

To visualize the differences between the groups, Fig. 2 provides women's and men's ratings for the items across the samples. Spanish, Chilean, and French women show high agreement in their ratings across items, but women from the USA present a different pattern. Furthermore, Fig. 2 illustrates that the Bought Jewelry item is an oddity for the non-USA samples, which show a steep drop below 0 for only this item. Figure 2 also shows that men's ratings are more homogeneous across the four cultures than women's ratings.

\section{Discussion}

We attempted to replicate the finding that male participants infer greater sexual intent on the part of a hypothetical woman than female participants. The ratings collected in Spain, Chile, and France exhibited a similar, but smaller, sex difference compared to ratings from the USA. The explanation for the sex difference could be due to men overestimating women's sexual intentions, women understating their own sexual intentions, or a combination of these two (Perilloux and Kurzban 2015), a point to which we return, below.

As to the source of the cultural variation, while we cannot speak definitively to its source, we can offer some proposals based on considerations of the details of the patterns of data for each country. Among the French, the overall effect is driven by a single item, Bought Jewelry. This item, in contrast to items such as Held Hands, Compliment, or Kiss, specifies a behavior that is extremely rare, and even counter-normative, among newly dating French couples, according to our colleagues who have lived in France. In real French life, then, it is unlikely that misperception as a result of a woman buying a man jewelry occurs. Indeed, the fact that it is so rare might explain the difference in views by male and female participants; having little or no experience of it, they disagree about its meaning. Whatever the source of the disagreement, the observation that misperception is restricted to behavior that, in the first instance, rarely if ever occurs, leads to the inference that there is little difference in what men infer and what women intend for the behaviors that people in French society actually engage in. We take it, then, that we have gathered no
Fig. 1 Composite scores on dating behaviors scale by country. Note: USA data are from Perilloux and Kurzban (2015). Error bars depict $95 \%$ confidence intervals, and all sex differences were significant at $p<.05$

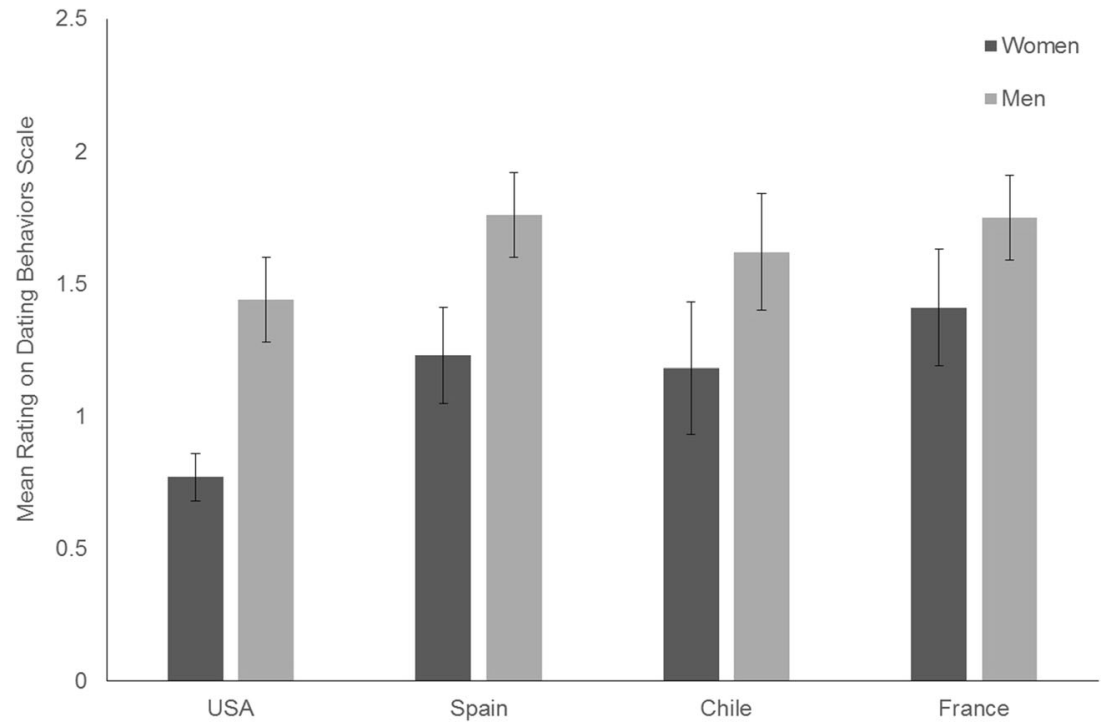


Table 1 Results of a MANOVA of sex differences by item
Fig. 2 Differences in item ratings across countries for women and men. Note: USA data come from Perilloux and Kurzban (2015). Error bars depict $95 \%$ confidence intervals

\begin{tabular}{|c|c|c|c|c|}
\hline Item & $\begin{array}{l}\text { USA } \\
F\left(\eta_{p}^{2}\right)\end{array}$ & $\begin{array}{l}\text { Spain } \\
F\left(\eta_{p}{ }^{2}\right)\end{array}$ & $\begin{array}{l}\text { Chile } \\
F\left(\eta_{p}^{2}\right)\end{array}$ & $\begin{array}{l}\text { France } \\
F\left(\eta_{p}{ }^{2}\right)\end{array}$ \\
\hline Held hands & $61.31 *(.11)$ & $0.40(.00)$ & $3.73(.04)$ & $2.37(.03)$ \\
\hline Compliment & $53.17 *(.10)$ & $2.82(.02)$ & $0.08(.00)$ & $1.13(.01)$ \\
\hline Bought jewelry & $94.61 *(.16)$ & $51.13 *(.24)$ & $30.09 *(.24)$ & $16.48 *(.17)$ \\
\hline Bought dinner & $62.45 *(.11)$ & $21.66 *(.12)$ & $16.13 *(.15)$ & $3.60(.04)$ \\
\hline Sent roses & $62.54 *(.11)$ & $6.04(.04)$ & $1.28(.01)$ & $5.88(.07)$ \\
\hline Cooked dinner & $34.70 *(.07)$ & $1.75(.01)$ & $0.03(.00)$ & $0.87(.01)$ \\
\hline Stared deeply & $24.86 *(.05)$ & $0.15(.00)$ & $2.38(.02)$ & $0.11(.00)$ \\
\hline Known forever & $3.09(.01)$ & $0.47(.00)$ & $0.16(.00)$ & $0.18(.00)$ \\
\hline Want to meet family & $3.69(.01)$ & $4.44(.03)$ & $0.65(.01)$ & $0.00(.00)$ \\
\hline Touched thigh & $26.47 *(.05)$ & $3.93(.02)$ & $7.68(.08)$ & $0.65(.01)$ \\
\hline Drink at apartment & $48.47 *(.09)$ & $15.81(.09)$ & $7.32(.07)$ & $0.03(.00)$ \\
\hline Kiss & $40.25 *(.08)$ & $9.29 *(.06)$ & $4.25(.04)$ & $0.43(.00)$ \\
\hline Said I love you & $8.66^{*}(.02)$ & $7.13(.04)$ & $1.03(.01)$ & $2.87(.03)$ \\
\hline Sex & $10.94 *(.02)$ & $27.81 *(.15)$ & $8.46(.08)$ & $0.46(.01)$ \\
\hline Spend life together & $1.75(.00)$ & $15.87 *(.09)$ & $5.15(.05)$ & $3.09(.04)$ \\
\hline
\end{tabular}

USA data are from Perilloux and Kurzban (2015). $\mathrm{USA}_{d f}=482, \operatorname{Spain}_{d f}=158$, Chile $_{d f}=93$, France $_{d f}=82$ ${ }^{*} p<.003$ (per Bonferroni correction), indicating that men rated the item significantly higher than women in that sample (there were no instances of women rating any item significantly higher than men)
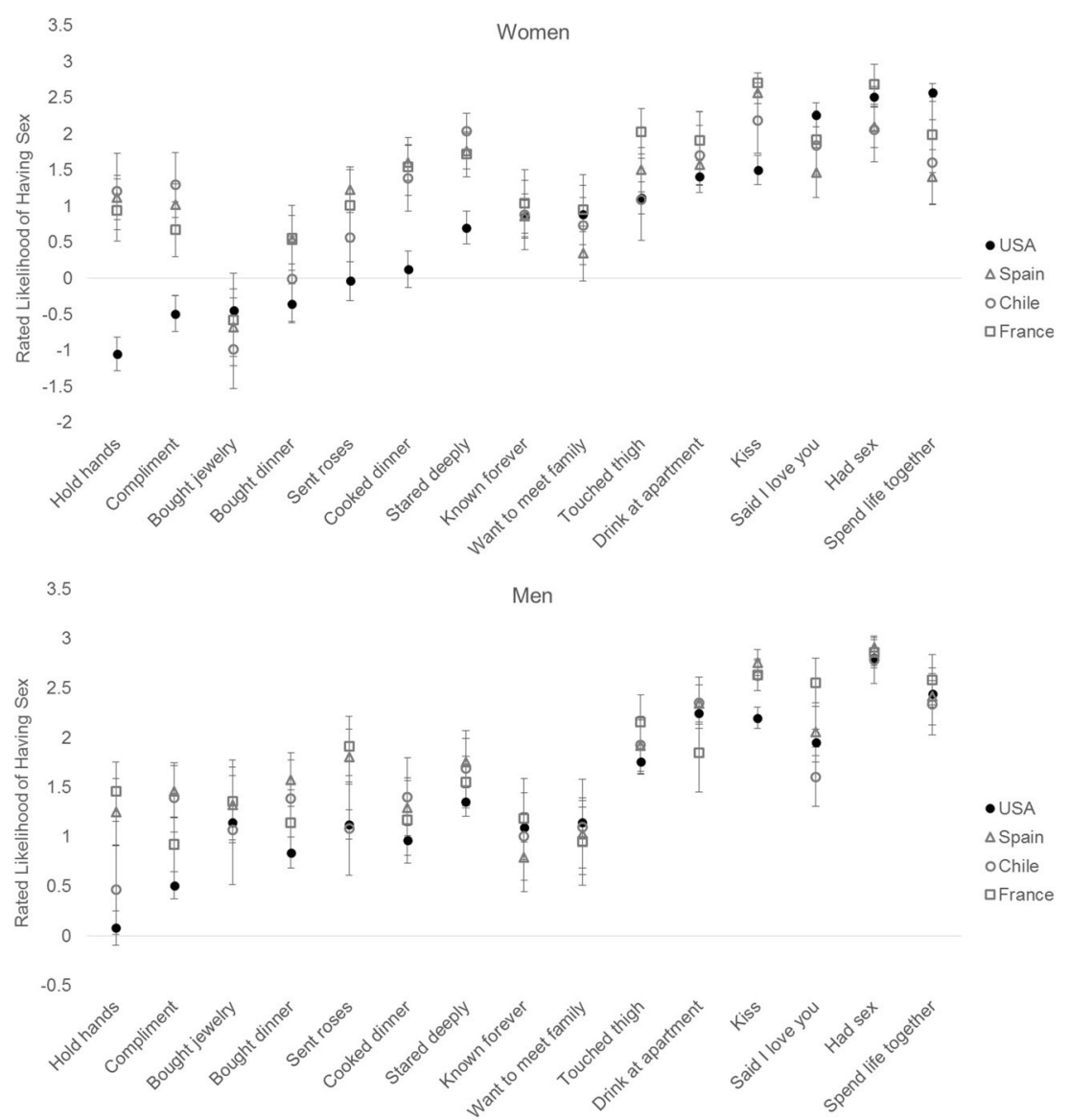
evidence that French men over-perceive the sexual intent of French women in their real lives.

Among the participants in the Spanish-speaking countries, Spain and Chile, the pattern we observe across the scale items falls somewhere between the USA sample and the French sample. As with the French sample, there is a substantial sex difference for the Bought Jewelry item. And, in parallel with France, our colleagues in these two countries echo the observation that women do not buy men jewelry early in the dating process, a view bolstered by some recent research in Chile that revealed that women are generally uninterested in displaying wealth to potential mates (Fernández et al. 2015); we therefore draw a similar conclusion. Still, among these samples, this item was not the only significant sex difference, and Bought Dinner, for instance, similarly shows a difference. It could be, then, that the sex difference found in the USA exists in these two countries, albeit in different, indeed, muted, form. We tentatively suggest that sexual misperception might be a less frequent occurrence in these countries than in the USA.

Further, recall that in the analysis of all eight groups (two sexes, four countries), we found that women from the USA stand out. To us, this points to the possibility — but of course does not show-that women from the USA are somehow different in how they respond to these items (though they were not significantly different from Chilean women). Still, the differences in the patterns observed across the items on the scale (Fig. 2) raise this possibility and suggests a direction for future research. If it does turn out that women from the USA are different in this respect from women elsewhere, conclusions from samples from the USA must be treated with special caution. For this reason, future studies could attempt to sample even more diverse cultures. Indeed, Bendixen (2014) recently replicated Haselton's (2003) study of memory for naturally occurring instances of apparent sexual interest misperception. Still, more samples would be useful in this regard.

Furthermore, we understand the original error management framework to imply that the effect should be general, in that men should tend to over-infer sexual interest from a large range of women's social and romantic behaviors. The present results suggest the possibility that misperception is a specific, rather than general, phenomenon, limited to a narrow band of behaviors rather than a wide swath of them. This, coupled with the finding for Bought Jewelry, suggests that this moment might constitute a good opportunity to consider updating the scale items with culturally normative behaviors. Additionally, many years have passed since the scale's initial construction during which dating and mating norms have changed, in some cases, dramatically. We look forward to scale development that reflects changes in norms and technologies.

Finally, there is the question of accuracy which applies to all studies of sexual interest ratings. The phenomenon of perceived sexual interest is particularly difficult to study because the truth of the matter - the probability of sex conditional on particular behaviors - remains unknown, and therefore, studies have only assessed differences in reports rather than the actual accuracy of participants' perceptions. Future studies could be designed to assess accuracy - these studies would be difficult to conduct, but have the potential to shed a lot of light on an area that has overwhelmingly relied upon (women's) self-report as reality.

Acknowledgments We would like to thank our translators Nico Benac and Leah Wilson.

\section{References}

Abbey, A. (1982). Sex differences in attributions for friendly behavior: do males misperceive females' friendliness? Journal of Personality and Social Psychology, 42, 830-838.

Abbey, A., Cozzarelli, C., McLaughlin, K., \& Harnish, J. (1987). The effect of clothing and dyad sex composition on perceptions of sexual intent: do women evaluate these cues differently. Journal of Applied Social Psychology, 17, 108-126.

Bendixen, M. (2014). Evidence of systematic bias in sexual over- and underperception of naturally occurring events: a direct replication of Haselton (2003) in a more gender-equal culture. Evolutionary Psychology, 12, 1004-1021.

DeSouza, E. R., Pierce, T., Zanelli, J. C., \& Hutz, C. (1992). Perceived sexual intent in the U.S. and Brazil as a function of nature of encounter, subjects' nationality, and gender. Journal of Sex Research, $29,251-260$.

Farris, C., Treat, T. A., Viken, R. J., \& McFall, R. M. (2008). Sexual coercion and the misperception of sexual intent. Clinical Psychology Review, 28, 48-66.

Fernández, A., Muñoz-Reyes, J. A., Dufey, M., Cid, V. \& Buccioni, G. (2015). Adaptación del cuestionario de componentes del valor de pareja al contexto chileno. Revista Iberoamericana de Diagnóstico y Evaluación Psicológica. (in press)

Haselton, M. G. (2003). The sexual overperception bias: evidence of a systematic bias in men from a survey of naturally occurring events. Journal of Research in Personality, 37, 34- 47.

Haselton, M. G., \& Buss, D. M. (2000). Error management theory: a new perspective on biases in cross-sex mind reading. Journal of Personality and Social Psychology, 78, 81-91.

Howell, E. C., Etchells, P. J., \& Penton-Voak, I. S. (2012). The sexual overperception bias is associated with sociosexuality. Personality and Individual Differences, 53, 1012-1016.

Perilloux, C., \& Kurzban, R. (2015). Do men misperceive women's sexual interest? Psychological Science, 26, 70-77.

Perilloux, C., Easton, J. A., \& Buss, D. M. (2012). The misperception of sexual interest. Psychological Science, 23, 146-151. 